\title{
Alexander invariants of ribbon tangles and planar algebras
}

\author{
By Celeste Damiani and Vincent Florens
}

(Received May 20, 2016)

(Revised Dec. 23, 2016)

\begin{abstract}
Ribbon tangles are proper embeddings of tori and cylinders in the 4-ball $B^{4}$, "bounding" 3-manifolds with only ribbon disks as singularities. We construct an Alexander invariant $\mathbf{A}$ of ribbon tangles equipped with a representation of the fundamental group of their exterior in a free abelian group $G$. This invariant induces a functor in a certain category $\mathbf{R} i b_{G}$ of tangles, which restricts to the exterior powers of Burau-Gassner representation for ribbon braids, that are analogous to usual braids in this context. We define a circuit algebra $\mathbf{C o b}_{G}$ over the operad of smooth cobordisms, inspired by diagrammatic planar algebras introduced by Jones [Jon99], and prove that the invariant $\mathbf{A}$ commutes with the compositions in this algebra. On the other hand, ribbon tangles admit diagrammatic representations, through welded diagrams. We give a simple combinatorial description of $\mathbf{A}$ and of the algebra $\mathbf{C} o b_{G}$, and observe that our construction is a topological incarnation of the Alexander invariant of Archibald [Arc10]. When restricted to diagrams without virtual crossings, A provides a purely local description of the usual Alexander poynomial of links, and extends the construction by Bigelow, Cattabriga and the second author [BCF15].
\end{abstract}

\section{Introduction.}

A ribbon torus link is a locally flat embedding of disjoint tori $S^{1} \times S^{1}$ in the ball $B^{4}$, bounding locally flat immersed solid tori $S^{1} \times D^{2}$ whose singular sets are finite numbers of ribbon disks. These elementary singularities are 4-dimensional analogues of the classical notion of ribbon introduced by Fox [Fox73]. Ribbon torus links are considered up to ambient isotopy; the fundamental groups of their complement and the derived Alexander modules provide topological invariants.

In this paper, we consider ribbon tangles that are ribbon proper embeddings of disjoint tori and cylinders in the ball $B^{4}$. The intersection of a given tangle $T$ with $\partial B^{4}=$ $S^{3}$ is a trivial link $L$. For a given free abelian group $G$, tangles are colored with a group homomorphism $\varphi: H_{1}\left(B^{4} \backslash T\right) \rightarrow G$. We construct an Alexander invariant A of colored ribbon tangles, lying in the exterior algebra of the homology $\mathbb{Z}[G]$-module $H_{1}^{\varphi}\left(S^{3} \backslash L\right)$, twisted by the morphism induced by $\varphi$, see Definition 2.4. This invariant coincides with the Alexander polynomial in the case of tangles with only two boundary components, see Proposition 2.6. The construction of $\mathbf{A}$ is based on the Alexander function introduced by Lescop [Les98]. The proof of the invariance and the main properties follow from algebraic and homological arguments developed in a paper by the second author and Massuyeau [FM15].

2010 Mathematics Subject Classification. Primary 57M25, 57M27, 57Q45.

Key Words and Phrases. Alexander polynomials, tangles, welded knots, planar algebras. 
Ribbon colored tangles can be splitted into morphisms in a category $\mathbf{R} i b_{G}$. We show that the invariant $\mathbf{A}$ induces a functor from $\mathbf{R} i b_{G}$ to the category of $\mathbb{Z}[G]$-graded modules, see Theorem 2.10. In the case of ribbon braids, that are the analogues of braids in this context, the functor coincides with the exterior powers of the ad-hoc colored Burau-Gassner representation, multiplied by a certain relative Alexander polynomial, see Proposition 2.9.

The multiplicativity of $\mathbf{A}$ fits naturally in the context of planar algebras, introduced by Jones [Jon99]. We construct an algebra $\mathbf{C o b}_{G}$ over the colored cobordisms operad, see Theorem 3.3. The cobordisms are smooth cylinder in $B^{4}$ with a finite collection of disjoint "small" balls removed, and the compositions are given by identification of some boundary components. We observe that cobordisms act on tangles and that $\mathbf{A}$ commutes with this action, see Theorem 3.4.

Ribbon knotted objects admit representations as broken surfaces via projections on the 3-dimensional space, and through welded diagrams which are representations in the 2-dimensional space. These representations were first introduced by Yajima [Yaj64] and Satoh [Sat00]. A complete description is given in the papers by Audoux, Bellingeri, Meilhan and Wagner [ABMW17], [Aud16]. This offers perspectives from both point of view: diagrams permit combinatorial computations of invariants of ribbon knotted objects, and ribbon knotted objects provide topological realizations of welded diagrams. Moreover, a presentation of the group of ribbon knotted objects is obtained from the diagrams by a Wirtinger type algorithm, see [ABMW17].

We give a diagrammatic description of the invariant $\mathbf{A}$, see Theorem 4.6 and show that it extends the multivariable Alexander polynomial of virtual tangles developed by Archibald and Bar-Natan. For futher details on these objects, see also [BNS13] and [Pol10]. The algebra $\mathbf{C o b} G$ appears as a topological incarnation of a circuit algebra of diagrams $\mathbf{W} e l d_{\mu}$, essentially introduced in [Arc10]. The compatibility of $\mathbf{A}$ with this structure allows local calculations in the diagram. If these diagrams do not have any virtual crossing, the construction holds for usual links, and we obtain a purely local description of the usual Alexander polynomial. This extends the construction of the Alexander representation by the second author, Bigelow and Cattabriga, see [BCF15].

Our construction arises in the context of defining generalizations of Alexander polynomials to tangle-like objects, in which we can include, in addition to [BCF15] concerning usual tangles and [Arc10], [Pol10] concerning virtual tangles, also the works of Cimasoni and Turaev, through Lagrangian categories [CT05], Bigelow [Big12] and Kennedy [Ken13] which studied diagrammatical invariants of usual tangles, Sartori [Sar15], who defined quantum invariants of framed tangles, and Zibrowius in [Zib16]. This last one in particular defines an invariant for usual tangles which consists in a finite set of Laurent polynomials, and states, without explicit calculation, that on usual tangles, one can calculate Archibald's invariant from his set of invariants and vice versa. However, nothing seems to appear in the literature about the 4-dimensional case of ribbon tangles.

In Section 2, we recall the definitions of ribbon tangles and construct the invariant $\mathbf{A}$. Section 3 is devoted to the circuit algebra $\mathbf{C o b}_{G}$ over the cobordism operad, and the properties of $\mathbf{A}$ with respect with the action of cobordisms on tangles. In Section 4, we describe the diagrammatic construction of $\mathbf{A}$ and a circuit algebra $\mathbf{W} e l d_{\mu}$ related 
to $\mathbf{C o b} b_{G}$. In Section 5, we compute some examples.

\section{The Alexander invariant A.}

In this section, $G$ is a free abelian group, and $R$ is the group ring $\mathbb{Z}[G]$.

\subsection{Ribbon tangles.}

Let $m$ be a positive integer and $X$ a submanifold of the $m$-dimensional ball $B^{m}$. An immersion $Y \subset X$ is locally flat if and only if it is locally homeomorphic to a linear subspace $\mathbb{R}^{k}$ in $\mathbb{R}^{m}$ for some $k \leq m$, except on $\partial X$ and/or $\partial Y$, where one of the $\mathbb{R}$ summands should be replaced by $\mathbb{R}_{+}$. An intersection $Y_{1} \cap Y_{2} \subset X$ is flatly transverse if it is locally homeomorphic to the intersection of two linear subspaces $\mathbb{R}^{k_{1}}$ and $\mathbb{R}^{k_{2}}$ in $\mathbb{R}^{m}$ for some positive integers $k_{1}, k_{2} \leq m$ except on $\partial X, \partial Y_{1}$ and/or $\partial Y_{2}$, where one of the $\mathbb{R}$ summands is replaced by $\mathbb{R}_{+}$.

An intersection $D=Y_{1} \cap Y_{2} \subset S^{4}$ is a ribbon disk if it is homeomorphic to the 2-disk and satisfies: $D \subset \stackrel{\circ}{Y}_{1}, \stackrel{\circ}{D} \subset \stackrel{\circ}{Y}_{2}$ and $\partial D$ is an essential curve in $\partial Y_{2}$. More details on ribbon knotted objects can be found in [ABMW17], [Aud16]. Denote $I=[0 ; 1]$.

Definition 2.1. Let $L$ be an oriented trivial link with $2 n$ components in $S^{3}=\partial B^{4}$. A ribbon tangle $T$ is a locally flat proper embedding in $B^{4}$ of oriented disjoint annuli $S^{1} \times I$ denoted $A_{1}, \ldots, A_{n}$ and disjoint tori $S^{1} \times S^{1}$ denoted $E_{1}, \ldots, E_{m}$ such that:

i) There exist locally flat immersed solid tori $F_{i}$ for $i=1, \ldots, m$ such that $\partial F_{i}=E_{i}$.

ii) $\partial A_{i} \subset L$ and the orientation induced by $A_{i}$ on $\partial A_{i}$ coincides with the given orientation of the two components of $L$.

iii) There exist $n$ locally flat immersed 3-balls $B_{i} \simeq B^{2} \times I$ such that, for all $i \in$ $\{1, \ldots, n\}$ :

$$
\partial B_{i}=A_{i} \cup_{\partial}\left(B^{2} \times\{0,1\}\right)
$$

iv) The singular set of the fillings $B_{i}$ and $F_{j}$ is composed by a finite number of ribbon disks.

A $G$-colored ribbon tangle is a pair $(T, \varphi)$ where $T$ is a ribbon tangle with complement $X_{T}=B^{4} \backslash T$, equipped with a group homomorphism $\varphi: H_{1}\left(X_{T}\right) \rightarrow G$.

\subsection{Definition of the Alexander invariant $\mathbf{A}$.}

Let $(X, Y)$ be a pair of topological spaces. Denote $p: \hat{X} \rightarrow X$ the maximal abelian cover. For a ring homomorphism $\varphi: \mathbb{Z}\left[H_{1}(X)\right] \rightarrow R$, we define the twisted chain complex

$$
C^{\varphi}(X, Y)=C\left(\hat{X}, p^{-1}(Y)\right) \otimes_{\mathbb{Z}\left[H_{1}(X)\right]} R
$$

whose homology is denoted $H_{*}^{\varphi}(X, Y ; R)$, or simply $H_{*}^{\varphi}(X, Y)$.

Let $(T, \varphi)$ be a $G$-colored ribbon tangle. The homomorphism $\varphi$ extends to a ring homomorphism $\varphi: \mathbb{Z}\left[H_{1}\left(X_{T}\right)\right] \rightarrow R$. For the rest of this section, we set $H=H_{1}^{\varphi}\left(X_{T}, *\right)$.

Proposition 2.2. The $R$-module $H$ admits a presentation with deficiency $n$. 
We postpone the proof of Proposition 2.2 to the end of Section 4.1. Consider a presentation of $H$ of the form:

$$
H=\left\langle\gamma_{1}, \ldots, \gamma_{n+q} \mid r_{1}, \ldots, r_{q}\right\rangle .
$$

Let $\Gamma$ be the free $R$-module generated by $\left\langle\gamma_{1}, \ldots, \gamma_{n+q}\right\rangle$ : the relators $r_{1}, \ldots, r_{q}$ are words in these generators in $\Gamma$. Let us denote $r=r_{1} \wedge \cdots \wedge r_{q}$ and $\gamma=\gamma_{1} \wedge \cdots \wedge \gamma_{n+q}$. The Alexander function $\mathcal{A}_{T}^{\varphi}: \wedge^{n} H \rightarrow R$ is the $R$-linear map defined by

$$
r \wedge \tilde{u}=\mathcal{A}_{T}^{\varphi}(u) \cdot \gamma
$$

for all $u=u_{1} \wedge \cdots \wedge u_{n} \in \wedge^{n} H$, where $\tilde{u}_{1}, \cdots, \tilde{u}_{n}$ are arbitrary lifts in $\Gamma$ and $\tilde{u}=$ $\tilde{u}_{1} \wedge \cdots \wedge \tilde{u}_{n}$. Different $n$-deficient presentations will give rise to Alexander functions that differ only by multiplication by a unit in $R$. Note that if $H$ is free of rank $n$, then $\mathcal{A}_{T}^{\varphi}$ is a volume form.

Let us consider the $q \times(q+n)$ matrix defined by the presentation of $H$. If one adds to this matrix the row vectors giving $u_{1}, \ldots, u_{n}$ in the generators $\gamma_{1}, \ldots, \gamma_{q+n}$, then $\mathcal{A}_{T}^{\varphi}(u)$ is the determinant of the resulting $(q+n) \times(q+n)$ matrix.

ExAmple 2.3. Suppose that $G$ has rank 2 and is generated by $t_{1}, t_{2}$. Consider the module $H$ whose presentation has generators $\gamma_{1}, \ldots, \gamma_{4}$ and two relations given by the matrix:

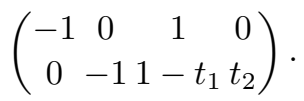

The values of the Alexander function $\mathcal{A}: \wedge^{2} H \rightarrow R$ are

$$
\begin{gathered}
\mathcal{A}\left(\gamma_{1} \wedge \gamma_{2}\right)=t_{2}, \mathcal{A}\left(\gamma_{1} \wedge \gamma_{3}\right)=0, \mathcal{A}\left(\gamma_{1} \wedge \gamma_{4}\right)=1, \\
\mathcal{A}\left(\gamma_{2} \wedge \gamma_{3}\right)=-t_{2}, \mathcal{A}\left(\gamma_{2} \wedge \gamma_{4}\right)=1-t_{1}, \mathcal{A}\left(\gamma_{3} \wedge \gamma_{4}\right)=1
\end{gathered}
$$

Let $H_{\partial}=H_{1}^{\varphi}\left(S^{3} \backslash L, *\right)$, which is the free $R$-module of rank $2 n$, generated by the meridians of $L$. Let $m_{\partial}: H_{\partial} \rightarrow H$ be induced by the inclusion map $S^{3} \backslash L \hookrightarrow X_{T}$. For short, for a given $z \in \wedge^{n} H_{\partial}$, we use the notation $m_{\partial} z$ for $\wedge^{n} m_{\partial}(z)$.

Definition 2.4. The element $\mathbf{A}(T, \varphi)$ of $\wedge^{n} H_{\partial}$ is the (colored) isotopy invariant defined by the following property:

$$
\forall z \in \wedge^{n} H_{\partial}, \mathcal{A}_{T}^{\varphi}\left(m_{\partial} z\right)=\omega_{\partial}(\mathbf{A}(T, \varphi) \wedge z)
$$

where $\omega_{\partial}$ is a volume form on $H_{\partial}$.

\subsection{The Alexander polynomial of a $(1-1)$-tangle.}

Given a finitely generated $R$-module $H$, and $k \geq 0$, the $k$-th Alexander polynomial of $H$ is the greatest common divisor of all minors of order $(m-k)$ in a $q \times m$ presentation matrix of $H$. This invariant of $H$, denoted $\Delta_{k}(H) \in R$, is defined up to multiplication by a unit of $R$. 
Definition 2.5. The Alexander polynomial $\Delta^{\varphi}(T) \in R$ of a $G$-colored ribbon tangle $(T, \varphi)$ is $\Delta_{0}\left(H_{1}^{\varphi}\left(X_{T}\right)\right)$.

Similarly to classical knot theory, $T$ is a $(1-1)$-tangle if $n=1$. The components of $T$ in $B^{4}$ consist of $m$ tori and a cylinder whose boundary is a 2-component trivial link $L$ in $S^{3}=\partial B^{4}$. Let $x_{1}$ and $x_{2}$ be the meridians of the components of $L$. Note that in $X_{T}$, both $x_{1}$ and $-x_{2}$ are homologous to the meridian $x$ of the cylinder. We use the same notations $x_{1}$ and $x_{2}$ for the homology classes of their lifts in $H_{1}^{\varphi}\left(S^{3} \backslash L, *\right)$.

Proposition 2.6. Let $(T, \varphi)$ be a G-colored $(1-1)$-tangle, such that $\varphi$ is not trivial. Denote $t=\varphi(x)$ and let $r$ be the rank of $\varphi\left(H_{1}\left(X_{T}\right)\right)$. Then the element $\mathbf{A}(T, \varphi)$ of $H_{\partial}$ is given by

$$
\mathbf{A}(T, \varphi)= \begin{cases}(t-1) \Delta^{\varphi}(T) \cdot\left(x_{1}-x_{2}\right) & \text { if } r \geq 2 \\ \Delta^{\varphi}(T) \cdot\left(x_{1}-x_{2}\right) & \text { if } r=1 .\end{cases}
$$

It is worth noticing that, up to a unit in $R$, the result is independent of the order chosen on the components of $L$. For the reader's convenience, we give a short proof of Proposition 2.6. More detailed arguments can be found in [FM15, Section 3].

Proof. Denote $H=H_{1}^{\varphi}\left(X_{T}, *\right)$. From the long exact sequence of the pair $\left(X_{T}, *\right)$ :

$$
0 \longrightarrow H_{1}^{\varphi}\left(X_{T}\right) \longrightarrow H \longrightarrow H_{0}^{\varphi}(*) \longrightarrow H_{0}^{\varphi}\left(X_{T}\right) \longrightarrow 0
$$

we deduce that $\operatorname{Tors} H_{1}^{\varphi}\left(X_{T}\right) \simeq \operatorname{Tors} H$. Moreover, $\operatorname{rk} H=\operatorname{rk} H_{1}^{\varphi}\left(X_{T}\right)+1$. This implies that

$$
\Delta^{\varphi}(T)=\Delta_{1}(H)
$$

Let $A$ be the matrix of a presentation of $H=\left\langle\gamma_{1}, \ldots, \gamma_{q+1} \mid r_{1}, \ldots, r_{q}\right\rangle$, and $\mathcal{A}^{\varphi}$ be the related Alexander function. We have

$$
\forall z_{1}, \ldots, z_{q+1} \in R, \mathcal{A}^{\varphi}\left(z_{1} \gamma_{1}+\cdots+z_{q+1} \gamma_{q+1}\right)=\sum_{i=1}^{q+1} \operatorname{det}\left(A_{i}\right) z_{i},
$$

where $A_{i}$ is $A$ with the $i$-th column removed. Hence,

$$
\Delta_{1}(H)=\operatorname{gcd} \mathcal{A}^{\varphi}(H)=\operatorname{gcd}\{\varphi(h) ; h \in H\} .
$$

If $\mathcal{A}^{\varphi}=0$, then $\Delta_{\varphi}(T)=0$. Consider now the connecting homomorphism $\partial_{*}: H \rightarrow$ $H_{0}^{\varphi}(*) \simeq R$. If $\mathcal{A}^{\varphi} \neq 0$, then $\operatorname{rank}(H)=1$ and two linear maps $H \otimes_{R} \mathbb{Q} R \rightarrow \mathbb{Q} R$ are linearly dependent, where $\mathbb{Q} R$ is the fraction field of $R$. Then there exist elements $P, Q$ in $R$ such that for each $h \in H, \mathcal{A}^{\varphi}(h)=(P / Q) \partial_{*}(h)$. Hence

$$
\mathcal{A}^{\varphi}(h)=\Delta^{\varphi}(T) \cdot \frac{\partial_{*}(h)}{\operatorname{gcd} \partial_{*}(H)} .
$$


For a loop $\gamma$ based in $*$ with lift $\hat{\gamma}$, one has $\partial_{*} \hat{\gamma}=\varphi(\gamma)-1$. Hence, $\operatorname{gcd} \partial_{*}(H)$ is equal to 1 if $r \geq 2$ and is equal to $t-1$ if $r=1$. We deduce that for all $h \in H$

$$
\mathcal{A}^{\varphi}(h)= \begin{cases}(t-1) \Delta^{\varphi}(T) & \text { if } r \geq 2, \\ \Delta^{\varphi}(T) & \text { if } r=1 .\end{cases}
$$

Let $\omega_{\partial}$ be the volume form on $H_{\partial}$ relative to the choice of the meridians $x_{1}, x_{2}$. By definition, $\mathbf{A}(T, \varphi)$ verifies $\mathcal{A}_{T}^{\varphi}\left(m_{\partial} z\right)=\omega_{\partial}(\mathbf{A}(T, \varphi) \wedge z)$. Since $m_{\partial}\left(x_{1}\right)=x$ and $m_{\partial}\left(x_{2}\right)=$ $-x$, we obtain the result from

$$
\mathcal{A}^{\varphi}(x)=\omega_{\partial}\left(\mathbf{A}(T, \varphi) \wedge x_{1}\right)=-\omega_{\partial}\left(\mathbf{A}(T, \varphi) \wedge x_{2}\right) .
$$

\subsection{The Burau functor.}

We begin with defining ribbon cobordisms in $B^{3} \times I$. Let $L_{0}$ and $L_{1}$ be two trivial links in the interior of $B^{3}$. Assign a sign to each component of the links. A ribbon cobordism from $L_{0}$ to $L_{1}$ is a collection $S$ of ribbon annuli and tori in $B^{3} \times I$ whose boundaries are $L_{0}$ in $B^{3} \times\{0\}$ and $L_{1}$ in $B^{3} \times\{1\}$, with according signs. Two cobordisms are equivalent if there is an ambient isotopy fixing the boundary circles $L_{0}$ and $L_{1}$.

The category $\mathcal{R} i b$ of ribbon cobordisms is the category whose: objects are sequences $\varepsilon$ of signs \pm 1 of length $n$, corresponding to trivial links with $n$ components in $B^{3}$, such that $\varepsilon$ is a sequence of signs affected to the components; morphisms $\varepsilon_{0} \rightarrow \varepsilon_{1}$ are the equivalence classes of ribbon cobordisms between $L_{0}$ and $L_{1}$. The degree of a morphism $\varepsilon_{0} \rightarrow \varepsilon_{1}$ is $\delta=\left(n_{1}-n_{0}\right) / 2$. The composition $S \circ S^{\prime}$ of two ribbon cobordisms $S$ and $S^{\prime}$ in $\mathcal{R} i b$ is defined by identifying $\left(B^{3} \times\{1\}, S^{\prime}\right)$ to $\left(B^{3} \times\{0\}, S\right)$.

The category $\mathcal{R} i b$ can be refined to the category of colored ribbon cobordisms $\mathcal{R} i b_{G}$. An object is a pair $(\varepsilon, \varphi)$, where $\varphi: H_{1}\left(B^{3} \backslash L\right) \rightarrow G$. A morphism $\left(\varepsilon_{0}, \varphi_{0}\right) \rightarrow\left(\varepsilon_{1}, \varphi_{1}\right)$ is a pair $(S, \varphi)$ such that $\varphi \circ m_{i}=\varphi_{i}$ for $i=0,1$, if $m_{i}$ are induced by the inclusions $X_{L_{i}} \hookrightarrow$ $X_{S}$, where $X_{L_{i}}=\left(B^{3} \times\{i\}\right) \backslash L_{i}$ and $X_{S}=\left(B^{3} \times I\right) \backslash S$.

Let $\operatorname{grMod}_{G}$ be the category of $\mathbb{Z}$-graded $R$-modules, whose morphisms are graded $R$-linear maps of arbitrary degree, up to multiplication by an element of $\pm G$. We now define a projective functor $\rho: \mathcal{R} i b_{G} \longrightarrow \operatorname{gr} \mathcal{M o d}_{G}$, which will be called the Burau functor.

We fix a base point $*$ in $B^{3}$. Given a trivial link $L$ with $n$ components in $B^{3}$, and a homomorphism $\varphi: H_{1}\left(B^{3} \backslash L\right) \rightarrow G$, we denote by $M$ the free $R$-module of $H_{1}^{\varphi}\left(X_{L}, * ; R\right)$ of rank $n$. In particular, for $i=0,1$, taken $L_{i}$ as before on $B^{3} \times\{i\}$, we denote by $M_{i}$ the free $R$-module $H_{1}^{\varphi_{i}}\left(X_{L_{i}}, * ; R\right)$ of rank $n_{i}$. The map

$$
\rho: \mathcal{R i b}_{G} \rightarrow \operatorname{grMod}_{G}
$$

is defined by the following construction. Let $(\varepsilon, \varphi)$ be an object of $\mathcal{R} i b_{G}$, corresponding to a trivial link $L$. The image by $\rho$ of $(\varepsilon, \varphi)$ is

$$
\rho(\varepsilon, \varphi)=\wedge M
$$

the exterior algebra of the free $R$-module $M$. To a morphism $(S, \varphi):\left(\varepsilon_{0}, \varphi_{0}\right) \rightarrow\left(\varepsilon_{1}, \varphi_{1}\right)$, we associate a $R$-linear map 


$$
\rho(S, \varphi): \wedge M_{0} \longrightarrow \wedge M_{1}
$$

of degree $\delta$ as follows. Let $J$ be the interval in $\partial B^{3} \times I$, which connects the base points of the bottom and top balls. By Proposition 2.2, the $R$-module $H_{1}^{\varphi}\left(X_{S}, J ; R\right)$ admits a presentation with deficiency $d=\left(n_{0}+n_{1}\right) / 2$. Consider the Alexander function $\mathcal{A}_{S}^{\varphi}: \wedge^{d} H_{1}^{\varphi}\left(X_{S}, J ; R\right) \rightarrow R$. For any integer $k \geq 0$, the image $\rho(S, \varphi)(x) \in \wedge^{k+\delta} M_{1}$ of any $x \in \wedge^{k} M_{0}$ is defined by the following property

$$
\forall y \in \wedge^{d-k} M_{1}, \mathcal{A}_{S}^{\varphi}\left(\wedge^{k} m_{0}(x) \wedge \wedge^{d-k} m_{1}(y)\right)=\omega_{1}(\rho(S, \varphi)(x) \wedge y),
$$

where $\omega_{1}$ is a volume form on $M_{1}$.

TheOREm 2.7. The map $\rho$ is a degree preserving functor $\mathcal{R} i b_{G} \rightarrow \operatorname{grMod}_{G}$.

The proof of Theorem 2.7 follows word by word the proof of [FM15, Theorem I]. Note that the deficiency of the presentation of $H_{1}^{\varphi}\left(X_{S}, J ; R\right)$ depends only on $n_{0}$ and $n_{1}$.

A $G$-colored ribbon tube $(S, \varphi)$ is a morphism such that the inclusions $m_{0}$ and $m_{1}$ induce isomorphisms in homology (with integer coefficients). Ribbon tubes are analogous to string links; the links $L_{0}$ and $L_{1}$ have the same number of components, and $S$ has no toric component. For a fixed $\varphi$, the set of $G$-colored ribbon tubes forms a monoid $\mathcal{T}_{\varphi}$. Following [KLW01, Proposition 2.1], one proves that $m_{0}$ and $m_{1}$ induce isomorphisms

$$
\left(m_{i}\right)_{*}: H^{\varphi}\left(X_{L_{i}} ; Q R\right) \longrightarrow H^{\varphi}\left(X_{S} ; Q R\right), \text { for } i=0,1
$$

where $Q R$ is the quotient field of $R$. Set $H^{\varphi}$ to be $H^{\varphi}\left(X_{L_{0}} ; Q R\right)=H^{\varphi}\left(X_{L_{1}} ; Q R\right)$. Set $L=L_{0}=L_{1}$. The composition $m_{1}^{-1} \circ m_{0}$ is an automorphism of $H^{\varphi}\left(X_{L} ; Q R\right)$.

DeFinition 2.8. The colored Burau representation is the monoid homomorphism

$$
r^{\varphi}: \mathcal{T}_{\varphi} \rightarrow \operatorname{Aut}\left(H^{\varphi}\right)
$$

Let $\Delta^{\varphi}\left(X_{T}, X_{L}\right)=\Delta_{0}\left(H_{1}^{\varphi}\left(X_{T}, X_{L} ; R\right)\right)$ be the Alexander polynomial of the pair $\left(X_{T}, X_{L}\right)$, see Section 2.3 .

Proposition 2.9. For any $G$-colored ribbon tube $(S, \varphi) \in \mathcal{T}_{\varphi}$, we have

$$
\rho(S, \varphi)=\Delta^{\varphi}\left(X_{T}, X_{L}\right) \cdot \wedge r^{\varphi}: \wedge H^{\varphi} \rightarrow \wedge H^{\varphi} .
$$

In particular, if the ribbon tube $(S, \varphi)$ is monotone with respect to the coordinate in $I$ in $B^{4} \times I$, it is called a ribbon braid (see [ABMW17], [Dam17]). In this case $\Delta^{\varphi}\left(X_{T}, X_{L}\right)=1$ and $\rho(S, \varphi)$ coincide with the exterior powers of $r^{\varphi}$. The proof of Proposition 2.9 can be obtained similarly to [FM15, Proposition 7.2], or in the monotone case, to [BCF15, Section 3.1].

Let $(T, \varphi)$ be a $G$-colored ribbon tangle in $B^{4}$. Let $L=L_{0} \cup L_{1}$ be a splitting of $L$ into two disjoint (trivial) links, and let $B_{0}$ and $B_{1}$ be two 3 -balls such that

$$
S^{3}=B_{0} \cup_{S^{2} \times\{0\}} S^{2} \times[0 ; 1] \cup_{S^{2} \times\{1\}} B_{1}
$$


and $L_{i} \subset B_{i}$ for $i=0,1$. Let $\varphi_{i}$ be induced by $\varphi$ on $H_{1}\left(B_{i} \backslash L_{i}\right)$, and $\varepsilon_{i}$ be sequences of signs according to the co-orientations of the components of $T$. Then $(T, \varphi)$ is splitted as a morphism $(\tilde{T}, \varphi):\left(\varepsilon_{0}, \varphi_{0}\right) \rightarrow\left(\varepsilon_{1}, \varphi_{1}\right)$. Note that $2 n=n_{0}+n_{1}$.

TheOREM 2.10. Let $(T, \varphi)$ be a G-colored ribbon tangle, and $(\tilde{T}, \varphi):\left(\varepsilon_{0}, \varphi_{0}\right) \rightarrow$ $\left(\varepsilon_{1}, \varphi_{1}\right)$ be a splitting of $(\mathcal{T}, \varphi)$ in $\mathcal{R} i b_{G}$. There is an isomorphism, well-defined up to a unit in $R$,

$$
\wedge^{n} H_{\partial} \rightarrow \operatorname{Hom}_{R}\left(\wedge M_{0}, \wedge M_{1}\right)
$$

sending $\mathbf{A}(T, \varphi)$ to $\oplus_{k}(-1)^{k\left(n_{0}-k\right)} \rho_{k}(\tilde{T}, \varphi)$, where $\rho_{k}$ is the $k$-component of $\rho$.

An explicit example is given in Remark 5.1.

Proof. The decomposition $H_{\partial}=M_{0} \oplus M_{1}$ induces a natural isomorphism

$$
\wedge^{n} H_{\partial} \simeq \bigoplus_{k=0}^{n}\left(\wedge^{k} M_{0} \otimes \wedge^{n-k} M_{1}\right) .
$$

The element $\mathbf{A}(T, \varphi) \in \wedge^{n} H_{\partial}$ decomposes as $\sum_{k} \mathbf{A}_{k}(T, \varphi)$, where $\mathbf{A}_{k}(T, \varphi) \in \wedge^{k} M_{0} \otimes$ $\wedge^{n-k} M_{1}$. Suppose now that $k$ is fixed; the element $\mathbf{A}_{k}(T, \varphi)$ might not be decomposable. There exist a finite sequence of element $A_{0}^{l} \in \wedge^{k} M_{0}$ and $A_{1}^{l} \in \wedge^{n-k} M_{1}$ (depending on $k$, and $(T, \varphi))$ such that $\mathbf{A}_{k}(T, \varphi)=\sum_{l} A_{0}^{l} \otimes A_{1}^{l}$. Let $\omega_{0}$ be a volume form $\wedge^{n_{0}} M_{0} \rightarrow R$. There is an isomorphism

$$
\wedge^{k} M_{0} \otimes \wedge^{n-k} M_{1} \simeq \operatorname{Hom}_{R}\left(\wedge^{n_{0}-k} M_{0}, \wedge^{n-k} M_{1}\right)
$$

sending $\mathbf{A}_{k}(T, \varphi)$ to

$$
x \mapsto \sum_{l} \omega_{0}\left(x \wedge A_{0}^{l}\right) \cdot A_{1}^{l}
$$

We now show that this morphism coincides with $\rho_{n_{0}-k}(\tilde{T}, \varphi)$. Let $x \in \wedge^{n_{0}-k} M_{0}$. Note that we have $n_{0}-k+\delta=n-k$ and the morphism has degree $\delta$. Consider a volume form $\omega_{1}: \wedge^{n_{1}} M_{1} \rightarrow R$ and the sum $\omega_{\partial}=\omega_{0} \otimes \omega_{1}: \wedge^{n} H_{\partial} \rightarrow R$. Let $\mathcal{A}^{\varphi}$ be the Alexander function related to a presentation of $H_{1}^{\varphi}\left(X_{T}, * ; R\right)$ of deficiency $n$. Since $m_{\partial}=m_{0} \oplus m_{1}$ we have, for all $y$ in $\wedge^{k+\delta} M_{1}$,

$$
\begin{aligned}
\mathcal{A}^{\varphi}\left(\wedge^{n_{0}-k} m_{0}(x) \wedge \wedge^{k+\delta} m_{1}(y)\right) & =\omega_{\partial}(\mathbf{A}(T, \varphi) \wedge x \wedge y) \\
& =\omega_{\partial}\left(\mathbf{A}_{k}(T, \varphi) \wedge x \wedge y\right) \\
& =\sum_{l} \omega_{0}\left(A_{0}^{l} \wedge x\right) \cdot \omega_{1}\left(A_{1}^{l} \wedge y\right) \\
& =\omega_{1}\left(\sum_{l} \omega_{0}\left(A_{0}^{l} \wedge x\right) \cdot A_{1}^{l} \wedge y\right) .
\end{aligned}
$$


Hence $\sum_{l} \omega_{0}\left(x \wedge A_{0}^{l}\right) \cdot A_{1}^{l}=(-1)^{k\left(n_{0}-k\right)} \rho_{n_{0}-k}(\tilde{T}, \varphi)(x)$. Note that if, for cosmetic reasons, one prefers that $\mathbf{A}_{k}(T, \varphi)$ induces $(-1)^{k\left(n_{0}-k\right)} \rho_{k}(\tilde{T}, \varphi)(x)$ instead, one might compose with Hodge duality $\wedge^{k} M_{0} \rightarrow \wedge^{n_{0}-k} M_{0}$.

\section{The circuit algebra of colored cobordisms.}

In this section, we introduce the circuit algebra $\mathrm{Cob}_{G}$. The algebraic structure is inspired by [Jon99], [Pol10], see also [Ken13], [Arc10].

Definition 3.1. Let $B=B_{0}$ be a 4 -ball and $B_{1}, \ldots, B_{p}$ be disjoint 4-balls in the interior of $B$. For every $i \in\{0, \ldots, p\}$, let $L_{i}$ (with $L=L_{0}$ ) be a trivial oriented link with $2 n_{i}\left(n=n_{0}\right)$ components in $S_{i}^{3}=\partial B_{i}$ (with $\left.S^{3}=S_{0}^{3}\right)$. A cobordism $C$ is a disjoint union of locally flat proper embedded annuli in $B \backslash\left\{\stackrel{\circ}{B}_{1}, \ldots, \stackrel{\circ}{B}_{p}\right\}$, whose boundary are the links $L_{i}$, with the conditions of Definition 2.1 but without singularities.

Definition 3.2. Let $C^{\prime}$ and $C^{\prime \prime}$ be two cobordisms such that $B_{i}^{\prime}$ is a ball of $C^{\prime}$ with $n_{i}^{\prime}=n^{\prime \prime}$. The composition $C^{\prime} \circ_{i} C^{\prime \prime}$ is the cobordism obtained with the identification of $B^{\prime \prime}=B_{0}^{\prime \prime}$ with $B_{i}^{\prime}$.

As in the previous section, $G$ is a fixed free abelian group with group ring $R$. A $G$ colored cobordism is a pair $(C, \varphi)$ where $C$ is a cobordism with complement $X_{C}$, equipped with a group homomorphism $\varphi: H_{1}\left(B^{4} \backslash C\right) \rightarrow G$. The orientation-preserving diffeomorphism classes of $G$-colored cobordisms with composition of compatible cobordisms, form an operad denoted $\mathcal{C}_{G}$.

Let $\mathcal{H o m}_{G}$ be the operad of tensor powers of $R$-modules and $R$-linear applications, considered up to an element of $\pm G$. The composition in $\mathcal{H o m}_{G}$ is induced by the usual composition of maps. We construct the circuit algebra $\mathrm{Cob}_{G}$ as a morphism of operads from $\mathcal{C}_{G}$ to $\mathcal{H o m}_{G}$.

Let $(L, \varphi)$ be a $G$-colored oriented trivial link with $k$ components in $S^{3}$, with complement $X_{L}=S^{3} \backslash L$. The group homomorphism $\varphi: H_{1}\left(S^{3} \backslash L\right) \rightarrow G$ induces a ring homomorphism denoted also $\varphi: \mathbb{Z}\left[H_{1}\left(S^{3} \backslash L\right)\right] \rightarrow R$. Let $*$ be a base point on $S^{3}$. The $R$-module $H_{1}^{\varphi}\left(S^{3} \backslash L, * ; R\right)$ is free of rank $k$, generated by the meridians of $L$.

Let now $(C, \varphi)$ be a $G$-colored cobordism, with complement $X_{C}$. For $i=1, \ldots, p$, let $*$ and $*_{i}$ be base points in the boundary of $B_{i}$ and $J_{i}$ be intervals (whose interiors are disjoint, and disjoint from $C$ ) connecting $*$ to $*_{i}$. Note that the union of the $J_{i}$ is contractible. The homomorphism $\varphi$ induces a ring homomorphism $\mathbb{Z}\left[H_{1}\left(X_{C}\right)\right] \rightarrow R$ and also denoted $\varphi$. The inclusion $m_{i}: S_{i}^{3} \backslash L_{i} \hookrightarrow X_{T}$ induces $\varphi_{i}: \mathbb{Z}\left[H_{1}\left(S_{i}^{3} \backslash L_{i}\right)\right] \rightarrow R$. Set $H=H_{1}^{\varphi}\left(X_{C}, J\right), H_{\partial}=H_{1}^{\varphi}\left(S^{3} \backslash L, *\right)$, and $H_{\partial_{i}}=H_{1}^{\varphi_{i}}\left(S^{3} \backslash L_{i}, *_{i}\right)$ for $i=1, \ldots, p$. Note that $H$ is free of rank $r=n+n_{1}+\cdots+n_{p}$ and $H_{\partial_{i}}$ are free of rank $n_{i}$. Let $\omega_{C}^{\varphi}$ be a volume form $\omega_{C}^{\varphi}: \wedge^{r} H \rightarrow R$, and $\omega_{\partial}: \wedge^{2 n} H_{\partial} \rightarrow R$. For $i=1, \ldots, p$, we denote again $m_{i}: H_{\partial_{i}} \rightarrow H$ the map induced by the inclusion. Let $m: \otimes_{i} \wedge^{n_{i}} H_{\partial_{i}} \rightarrow \wedge^{n_{1}+\cdots+n_{p}} H$ be defined as $m=\wedge^{n_{1}} m_{1} \wedge \cdots \wedge \wedge^{n_{p}} m_{p}$.

To the cobordism $(C, \varphi)$ we associate

$$
\Upsilon_{C, \varphi}: \bigotimes_{i=1}^{p} \wedge^{n_{i}} H_{\partial_{i}} \rightarrow \wedge^{n} H_{\partial}
$$


such that, for $x \in \otimes_{i}\left(\wedge^{n_{i}} H_{\partial_{i}}\right)$,

$$
\omega_{C}^{\varphi}\left(m(x) \wedge m_{\partial}(y)\right)=\omega_{\partial}\left(\Upsilon_{C, \varphi}(x) \wedge y\right), \forall y \in \wedge^{n} H_{\partial} .
$$

$\operatorname{Cob}_{G}$ denotes the object that associates to a pair $(L, \varphi)$ the homology module $H_{1}^{\varphi}\left(S^{3} \backslash L, * ; R\right)$, and to each pair $(C, \varphi)$ the linear map $\Upsilon_{C, \varphi}$.

Theorem 3.3. $\mathrm{Cob}_{G}$ is a circuit algebra.

Proof. We show that $\Upsilon$ commutes with the composition of cobordisms. Let $\left(C^{\prime}, \varphi^{\prime}\right)$ and $\left(C^{\prime \prime}, \varphi^{\prime \prime}\right)$ be two cobordisms with

$$
\Upsilon_{C^{\prime}, \varphi^{\prime}}: \bigotimes_{k=1}^{p^{\prime}} \wedge^{k_{i}^{\prime}} H_{\partial_{k}^{\prime}} \rightarrow \wedge^{n^{\prime}} H_{\partial}^{\prime}, \quad \text { and } \quad \Upsilon_{C^{\prime \prime}, \varphi^{\prime \prime}}: \bigotimes_{l=1}^{p^{\prime \prime}} \wedge^{n_{l}^{\prime \prime}} H_{\partial_{l}^{\prime \prime}} \rightarrow \wedge^{n^{\prime \prime}} H_{\partial^{\prime \prime}}
$$

Let $\varphi$ be the coloring induced by $\varphi^{\prime}$ and $\varphi^{\prime \prime}$ on $C^{\prime} \circ_{i} C^{\prime \prime}$. Then, we have to prove that for all $u_{k} \in \wedge_{i}^{k} H_{\partial_{k}^{\prime}}$ with $k=1, \ldots, p^{\prime}$ and $k \neq i$ and all $v_{l}$ for $l=1, \ldots, p^{\prime \prime}$,

$$
\Upsilon_{C^{\prime} \circ_{i} C^{\prime \prime}, \varphi}\left(u_{1} \otimes \cdots \otimes\left(\otimes_{1}^{p^{\prime \prime}} v_{l}\right) \otimes \cdots \otimes u_{p^{\prime}}\right)=\Upsilon_{C^{\prime}, \varphi^{\prime}}\left(u_{1} \otimes \cdots \otimes \Upsilon_{C^{\prime \prime}, \varphi^{\prime \prime}}\left(\otimes_{1}^{p^{\prime \prime}} v_{l}\right) \otimes \cdots \otimes u_{p^{\prime}}\right) .
$$

Let $H^{\prime}=H_{1}^{\varphi^{\prime}}\left(X_{T^{\prime}}, J^{\prime}\right)$ and $H^{\prime \prime}=H_{1}^{\varphi^{\prime \prime}}\left(X_{T^{\prime \prime}}, J^{\prime \prime}\right)$ be the (free) homology modules of the exteriors of the cobordisms. Let $\alpha_{1}, \ldots, \alpha_{2 n^{\prime \prime}}$ be a basis of $H_{\partial^{\prime \prime}} \simeq H_{\partial_{i}^{\prime}}$. Consider presentations of $H^{\prime}$ and $H^{\prime \prime}$ :

$$
\begin{gathered}
H^{\prime \prime}=\left\langle m_{\partial^{\prime \prime}} \alpha_{1}, \ldots, m_{\partial^{\prime \prime}} \alpha_{2 n^{\prime \prime}}, \beta_{1}, \ldots, \beta_{k} \mid \rho_{1}, \ldots, \rho_{s}\right\rangle, \\
H^{\prime}=\left\langle m_{i}^{\prime} \alpha_{1}, \ldots, m_{i}^{\prime} \alpha_{2 n^{\prime \prime}}, \zeta_{1}, \ldots, \zeta_{l} \mid r_{1}, \ldots, r_{t}\right\rangle .
\end{gathered}
$$

Applying Mayer-Vietoris theorem to $X_{T}=X_{T^{\prime}} \cup X_{T^{\prime \prime}}$, we obtain that the (free) module $H$ is generated by

$$
m_{\partial^{\prime \prime}} \alpha_{1}, \ldots, m_{\partial^{\prime \prime}} \alpha_{2 n^{\prime \prime}}, m_{i}^{\prime} \alpha_{1}, \ldots, m_{i}^{\prime} \alpha_{2 n^{\prime \prime}}, \beta_{1}, \ldots, \beta_{k}, \zeta_{1}, \ldots, \zeta_{l}
$$

subject to the relations $\rho_{1}, \ldots, \rho_{s}, r_{1}, \ldots, r_{t}, m_{\partial^{\prime \prime}} \alpha_{1}-m_{i}^{\prime} \alpha_{1}, \ldots, m_{\partial^{\prime \prime}} \alpha_{2 n^{\prime \prime}}-m_{i}^{\prime} \alpha_{2 n^{\prime \prime}}$. Let $\omega^{\prime}$ and $\omega^{\prime \prime}$ be volume forms on $H^{\prime}$ and $H^{\prime \prime}$, and $\omega_{\partial^{\prime}}$ be the form on $H_{\partial^{\prime}}$. Let $\omega$ be the form on $H$ induced by $\omega^{\prime}$ and $\omega^{\prime \prime}$. For the computation below, we introduce the notation

$$
u \wedge_{i} v=u_{1} \wedge \cdots \wedge u_{i-1} \wedge\left(v_{1} \wedge \cdots \wedge v_{p^{\prime \prime}}\right) \wedge u_{i+1} \wedge \cdots \wedge u_{p^{\prime}}
$$

We want to show that, for all $y \in \wedge^{n^{\prime}} H_{\partial^{\prime}}$,

$$
\begin{aligned}
& \omega_{\partial^{\prime}}\left(\Upsilon_{C^{\prime} \circ C^{\prime \prime}}\left(u_{1} \otimes \cdots \otimes\left(\otimes_{1}^{p^{\prime \prime}} v_{l}\right) \otimes \cdots \otimes u_{p^{\prime}}\right) \wedge y\right) \\
& =\omega_{\partial^{\prime}}\left(\Upsilon_{C^{\prime}}\left(u_{1} \otimes \cdots \otimes \Upsilon_{C^{\prime \prime}}\left(\otimes_{1}^{p^{\prime \prime}} v_{l}\right) \otimes \cdots \otimes u_{p^{\prime}}\right) \wedge y\right) .
\end{aligned}
$$

We have

$$
\omega_{\partial^{\prime}}\left(\Upsilon_{C^{\prime} \circ C^{\prime \prime}}\left(u_{1} \otimes \cdots \otimes\left(\otimes_{1}^{p^{\prime \prime}} v_{l}\right) \otimes \cdots \otimes u_{p^{\prime}}\right) \wedge y\right) \cdot m_{\partial^{\prime \prime}} \alpha \wedge \beta \wedge m_{i}^{\prime} \alpha \wedge \zeta
$$




$$
\begin{aligned}
& =\omega\left(\left(m^{\prime} u \wedge_{i} m^{\prime \prime} v\right) \wedge m_{\partial^{\prime}} y\right) \cdot m_{\partial^{\prime \prime}} \alpha \wedge \beta \wedge m_{i}^{\prime} \alpha \wedge \zeta \\
& =\rho \wedge r \wedge\left(m_{\partial^{\prime \prime}} \alpha-m_{i}^{\prime} \alpha\right) \wedge\left(m^{\prime} u \wedge_{i} m^{\prime \prime} v\right) \wedge m_{\partial^{\prime}} y \\
& =\sum_{Q}(-1)^{|Q|} \epsilon_{Q} \cdot \rho \wedge r \wedge m_{\partial^{\prime \prime}} \alpha_{Q} \wedge m_{i}^{\prime} \alpha_{\bar{Q}} \wedge\left(m^{\prime} u \wedge_{i} m^{\prime \prime} v\right) \wedge m_{\partial^{\prime}} y
\end{aligned}
$$

where the sum is taken over all subsets $Q \subset\left\{1, \ldots, 2 n^{\prime \prime}\right\}$ of cardinal $n^{\prime \prime}$. The number $t$ of relations $r_{i}$ can be chosen arbitrarily to be even and $m_{\partial^{\prime \prime}} \alpha_{Q} \wedge m_{i}^{\prime} \alpha_{\bar{Q}} \wedge\left(m^{\prime} u \wedge_{i} m^{\prime \prime} v\right)$ coincides with $m_{\partial^{\prime \prime}} \alpha_{Q} \wedge m^{\prime \prime} v \wedge\left(m^{\prime} u \wedge_{i} m_{i}^{\prime} \alpha_{\bar{Q}}\right)$ up to a sign depending only on $p^{\prime \prime}$ and the $n_{i}^{\prime}$, the sum coincides up to a sign with

$$
\sum_{Q}(-1)^{|Q|} \epsilon_{Q} \cdot\left(\rho \wedge m_{\partial^{\prime \prime}} \alpha_{Q} \wedge m^{\prime \prime} v\right) \wedge\left(r \wedge\left(m^{\prime} u \wedge_{i} m_{i}^{\prime} \alpha_{\bar{Q}}\right) \wedge m_{\partial^{\prime}} y\right)
$$

whose summands are equal to

$$
(-1)^{|Q|} \epsilon_{Q} \cdot \omega^{\prime \prime}\left(m_{\partial^{\prime \prime}} \alpha_{Q} \wedge m^{\prime \prime} v\right) \cdot \omega^{\prime}\left(\left(m^{\prime} u \wedge_{i} m_{i}^{\prime} \alpha_{\bar{Q}}\right) \wedge m_{\partial^{\prime}} y\right) \cdot\left(m_{\partial^{\prime \prime}} \alpha \wedge \beta \wedge m_{i}^{\prime} \alpha \wedge \zeta\right) .
$$

It follows that, up to a sign,

$$
\begin{aligned}
& \omega_{\partial^{\prime}}\left(\Upsilon_{C^{\prime} \circ C^{\prime \prime}}\left(u_{1} \otimes \cdots \otimes\left(\otimes_{1}^{p^{\prime \prime}} v_{l}\right) \otimes \cdots \otimes u_{p^{\prime}}\right) \wedge y\right) \\
& =\sum_{Q}(-1)^{|Q|} \epsilon_{Q} \cdot \omega^{\prime \prime}\left(m_{\partial^{\prime \prime}} \alpha_{Q} \wedge m^{\prime \prime} v\right) \cdot \omega^{\prime}\left(\left(m^{\prime} u \wedge_{i} m_{i}^{\prime} \alpha_{\bar{Q}}\right) \wedge m_{\partial^{\prime}} y\right) \\
& =\omega^{\prime}\left(\sum_{Q}(-1)^{|Q|} \epsilon_{Q} \cdot \omega^{\prime \prime}\left(m_{\partial^{\prime \prime}} \alpha_{Q} \wedge m^{\prime \prime} v\right) \cdot\left(m^{\prime} u \wedge_{i} m_{i}^{\prime} \alpha_{\bar{Q}}\right) \wedge m_{\partial^{\prime}} y\right) \\
& =\omega^{\prime}\left(\sum_{Q}(-1)^{|Q|} \epsilon_{Q} \cdot \omega_{\partial^{\prime \prime}}\left(\Upsilon_{C^{\prime \prime}}\left(v_{1} \otimes \cdots \otimes v_{p^{\prime \prime}}\right) \wedge \alpha_{Q}\right) \cdot\left(m^{\prime} u \wedge_{i} m_{i}^{\prime} \alpha_{\bar{Q}}\right) \wedge m_{\partial^{\prime}} y\right) \\
& =\omega^{\prime}\left(\left(m^{\prime} u \wedge_{i}\left[\sum_{Q}(-1)^{|Q|} \epsilon_{Q} \cdot \omega_{\partial^{\prime \prime}}\left(\Upsilon_{C^{\prime \prime}}\left(v_{1} \otimes \cdots \otimes v_{p^{\prime \prime}}\right) \wedge \alpha_{Q}\right)\right] m_{i}^{\prime} \alpha_{\bar{Q}}\right) \wedge m_{\partial^{\prime}} y\right) \\
& =\omega^{\prime}\left(\left(m^{\prime} u \wedge_{i} m_{i}^{\prime}\left(\Upsilon_{C^{\prime \prime}}\left(v_{1} \otimes \cdots \otimes v_{p^{\prime \prime}}\right)\right)\right) \wedge m_{\partial}^{\prime} y\right) \\
& =\omega_{\partial^{\prime}}\left(\Upsilon_{C^{\prime}}\left(u_{1} \otimes \cdots \otimes \Upsilon_{C^{\prime \prime}}\left(\otimes_{1}^{p^{\prime \prime}} v_{l}\right) \otimes \cdots \otimes u_{p^{\prime}}\right) \wedge y\right) .
\end{aligned}
$$

\subsection{Action of cobordisms on ribbon tangles.}

Given a cobordism $C$ and a collection of ribbon tangles $T_{1}, \cdots, T_{p}$, one may create a new ribbon tangle, if $n\left(T_{i}\right)=n_{i}$ for all $i=1, \ldots, p$, by gluing each $T_{i}$ into the internal ball $B_{i}$ of $C$. The action of $G$-colored cobordisms on $G$-colored ribbon tangles is defined once the colorings coincide on the boundary components. The following theorem states that the invariant $\mathbf{A}$ respects the structure of circuit algebra $\mathrm{Cob}_{G}$.

THEOREM 3.4. Let $(T, \psi)$ be the $G$-colored ribbon tangle obtained by gluing the $G$-colored ribbon tangles $\left(T_{1}, \varphi_{1}\right), \cdots,\left(T_{p}, \varphi_{p}\right)$ to a $G$-colored cobordism $(C, \varphi)$. The following equality holds:

$$
\mathbf{A}(T, \psi)=\Upsilon_{C, \varphi}\left(\mathbf{A}\left(T_{1}, \varphi_{1}\right) \otimes \cdots \otimes \mathbf{A}\left(T_{p}, \varphi_{p}\right)\right) \in \wedge^{n} H_{\partial} .
$$

Proof. For $i=1, \cdots, p$, consider a presentation of $H_{T_{i}}=H_{1}^{\varphi_{i}}\left(X_{T_{i}}, *_{i}\right)$ of the 
form

$$
H_{T_{i}}=\left\langle m_{\partial_{i}} \gamma_{1}^{i}, \ldots, m_{\partial_{i}} \gamma_{2 n_{i}}^{i}, \beta_{1}^{i}, \ldots, \beta_{k_{i}}^{i} \mid \rho_{1}^{i}, \ldots, \rho_{s_{i}}^{i}\right\rangle
$$

and let $\mathcal{A}_{T_{i}}^{\varphi_{i}}$ be the Alexander function related to this presentation. Let $H_{C}=H_{1}^{\varphi}\left(X_{C}, J\right)$ be the free module, with volume form $\omega_{C}^{\varphi}$ associated to the presentation

$$
H_{C}=\left\langle m_{1} \gamma_{1}^{1}, \ldots, m_{1} \gamma_{2 n_{1}}^{1}, \ldots, m_{p} \gamma_{1}^{p}, \ldots, m_{p} \gamma_{2 n_{p}}^{p}, \alpha_{1}, \ldots, \alpha_{l} \mid r_{1}, \ldots, r_{t}\right\rangle .
$$

By successive Mayer-Vietoris arguments, the module $H_{T}=H_{1}^{\varphi}\left(X_{T}, *\right)$ admits a presentation with generators of the form $m_{\partial_{i}} \gamma_{j}^{i}, m_{i} \gamma_{j}^{i}$, for $j=1, \ldots, 2 n_{i}$ and $i=1, \ldots, p$, and $\beta_{1}^{i}, \ldots, \beta_{k_{i}}^{i}, \alpha_{1}, \ldots, \alpha_{l}$. They are subject to the relations $m_{\partial_{i}} \gamma_{j}^{i}-m_{i} \gamma_{j}^{i}$, for $j=1, \ldots, 2 n_{i}$ and $i=1, \ldots, p$, and $\rho_{1}^{i}, \ldots, \rho_{s_{i}}^{i}$ for $i=1, \ldots, p$ and $r_{1}, \ldots, r_{t}$. One has $\mathbf{A}(T, \psi)=$ $\Upsilon_{C, \varphi}\left(\mathbf{A}\left(T_{1}, \varphi_{1}\right) \otimes \cdots \otimes \mathbf{A}\left(T_{p}, \varphi_{p}\right)\right)$ if and only if

$$
\forall z \in \wedge^{n} H_{\partial}, \mathbf{A}(T, \psi) \wedge z=\Upsilon_{C, \varphi}\left(\mathbf{A}\left(T_{1}, \varphi_{1}\right) \otimes \cdots \otimes \mathbf{A}\left(T_{p}, \varphi_{p}\right)\right) \wedge z .
$$

By Equation (2.1), $\omega_{\partial}(\mathbf{A}(T, \psi) \wedge z)=\mathcal{A}_{T}^{\psi}\left(m_{\partial} z\right)$. For short, we write $\mathbf{A}\left(T_{i}\right)$ for $\mathbf{A}\left(T_{i}, \varphi_{i}\right)$, for $i=1, \ldots, p$. By definition of the Alexander function $\mathcal{A}_{T}^{\psi}$,

$$
\begin{aligned}
& \mathcal{A}_{T}^{\psi}\left(m_{\partial} z\right) \cdot m_{\partial_{*}} \gamma \wedge m_{*} \gamma \wedge \beta \wedge \alpha=\rho \wedge r \wedge\left(m_{\partial_{i}}^{i}-m_{i} \gamma^{i}\right) \wedge m_{\partial} z \\
& =\sum_{Q_{1}, \ldots, Q_{p}}(-1)^{|Q|} \epsilon_{Q} \cdot \rho \wedge r \wedge m_{\partial_{1}} \gamma_{Q_{1}}^{1} \wedge \cdots \wedge m_{\partial_{p}} \gamma_{Q_{p}}^{p} \wedge m_{1} \gamma_{\bar{Q}_{1}}^{1} \wedge \cdots \wedge m_{p} \gamma_{\bar{Q}_{p}}^{p} \wedge m_{\partial} z,
\end{aligned}
$$

where the sum is taken over the subsets $Q_{i} \subset\left\{1, \ldots, 2 n_{i}\right\}$ of cardinal $n_{i}$ (the other terms vanish). $\bar{Q}_{i}$ denotes the complement of $Q_{i},|Q|=n_{1}+\cdots+n_{p}$ and $\epsilon_{Q}$ the signature of the permutation $Q_{1} \cdots Q_{p} \bar{Q}_{1} \cdots \bar{Q}_{p}$, where the elements of $Q_{1} \cdots Q_{p}$ in increasing order are followed by the elements of $\bar{Q}_{1} \cdots \bar{Q}_{p}$ in increasing order. Moreover, we can decide arbitrarily that the number $t$ of relations in the presentation of $H_{C}$ is even, and get (3.3) to be equal to:

$$
\begin{aligned}
& \sum_{Q_{1}, \ldots, Q_{p}}(-1)^{|Q|} \epsilon_{Q} \cdot\left(\rho \wedge m_{\partial_{1}} \gamma_{Q_{1}}^{1} \wedge \cdots \wedge m_{\partial_{p}} \gamma_{Q_{p}}^{p}\right) \wedge\left(r \wedge m_{1} \gamma_{\bar{Q}_{1}}^{1} \wedge \cdots \wedge m_{p} \gamma_{\bar{Q}_{p}}^{p} \wedge m_{\partial} z\right) \\
& =\sum(-1)^{|Q|} \epsilon_{Q} \cdot\left(\rho \wedge m_{\partial_{1}} \gamma_{Q_{1}}^{1} \wedge \cdots \wedge m_{\partial_{p}} \gamma_{Q_{p}}^{p}\right) \cdot \omega_{C}^{\varphi}\left(m_{1} \gamma_{\bar{Q}_{1}}^{1} \wedge \cdots \wedge m_{p} \gamma_{\bar{Q}_{p}}^{p} \wedge m_{\partial} z\right) \\
& \cdot\left(m_{*} \gamma \wedge \alpha\right) \text {. }
\end{aligned}
$$

Moreover, since $s_{1}, \ldots, s_{p}$ can also be supposed even,

$$
\begin{aligned}
\rho \wedge m_{\partial_{1}} \gamma_{Q_{1}}^{1} \wedge \cdots \wedge m_{\partial_{p}} \gamma_{Q_{p}}^{p} & =\left(\rho^{1} \wedge m_{\partial_{1}} \gamma_{Q_{1}}^{1}\right) \wedge \cdots \wedge\left(\rho^{p} \wedge m_{\partial_{p}} \gamma_{Q_{p}}^{p}\right) \\
& =\mathcal{A}_{T_{1}}^{\varphi_{1}}\left(m_{\partial_{1}} \gamma_{Q_{1}}^{1}\right) \cdots \mathcal{A}_{T_{p}}^{\varphi_{p}}\left(m_{\partial_{p}} \gamma_{Q_{p}}^{p}\right) \cdot m_{\partial_{*}} \gamma \wedge \beta \\
& =\omega_{\partial_{1}}\left(\mathbf{A}\left(T_{1}\right) \wedge \gamma_{Q_{1}}^{1}\right) \cdots \omega_{\partial_{p}}\left(\mathbf{A}\left(T_{p}\right) \wedge \gamma_{Q_{p}}^{p}\right) \cdot m_{\partial_{*}} \gamma \wedge \beta .
\end{aligned}
$$

Then $\mathcal{A}_{T}^{\psi}\left(m_{\partial} z\right)$ coincides with the sum over $Q_{1} \cdots Q_{p}$ of the summands 


$$
\begin{aligned}
& (-1)^{|Q|} \epsilon_{Q} \cdot \omega_{\partial_{1}}\left(\mathbf{A}\left(T_{1}\right) \wedge \gamma_{Q_{1}}^{1}\right) \cdots \omega_{\partial_{p}}\left(\mathbf{A}\left(T_{p}\right) \wedge \gamma_{Q_{p}}^{p}\right) \cdot \omega_{C}^{\varphi}\left(m_{\bar{Q}_{1}} \gamma_{\bar{Q}_{1}}^{1} \wedge \cdots \wedge m_{\bar{Q}_{p}} \gamma_{\bar{Q}_{p}}^{p} \wedge m_{\partial} z\right) \\
& =\omega_{C}^{\varphi}\left(\sum(-1)^{|Q|} \epsilon_{Q} \cdot \omega_{\partial_{1}}\left(\mathbf{A}\left(T_{1}\right) \wedge \gamma_{Q_{1}}^{1}\right) \cdots \omega_{\partial_{p}}\left(\mathbf{A}\left(T_{p}\right) \wedge \gamma_{Q_{p}}^{p}\right)\right. \\
& \left.\quad \cdot m_{\bar{Q}_{1}} \gamma_{\bar{Q}_{1}}^{1} \wedge \cdots \wedge m_{\bar{Q}_{p}} \gamma_{\bar{Q}_{p}}^{p} \wedge m_{\partial} z\right)
\end{aligned}
$$

Note that for all $i=1, \ldots, p$ and $x \in \wedge^{n_{i}} H_{\partial_{i}}$, we have

$$
x=\sum_{\left|Q_{i}\right|=n_{i}} \epsilon_{\bar{Q}} \omega_{\partial_{i}}\left(x \wedge \gamma_{Q_{i}}^{i}\right) \cdot \gamma_{\bar{Q}_{i}}^{i}=\sum_{\left|Q_{i}\right|=n_{i}}(-1)^{\left|Q_{i}\right|} \epsilon_{Q_{i}} \omega_{\partial_{i}}\left(x \wedge \gamma_{Q_{i}}^{i}\right) \cdot \gamma_{\bar{Q}_{i}}^{i} .
$$

Moreover, $(-1)^{|Q|}=(-1)^{\left|Q_{1}\right|} \cdots(-1)^{\left|Q_{p}\right|}$ and $\epsilon_{Q} \epsilon_{Q_{1}} \cdots \epsilon_{Q_{p}}$ do not depend on $Q_{1}, \ldots, Q_{p}$ but only on $n_{1}=\left|Q_{1}\right|, \ldots, n_{p}=\left|Q_{p}\right|$. Hence, up to a sign

$$
\begin{aligned}
\mathcal{A}_{T}^{\psi}\left(m_{\partial} z\right) & =\omega_{C}^{\varphi}\left(m_{1} \mathbf{A}\left(T_{1}\right) \wedge \cdots \wedge m_{p} \mathbf{A}\left(T_{p}\right) \wedge m_{\partial} z\right) \\
& =\omega_{C}^{\varphi}\left(m\left(\mathbf{A}_{C}\left(\Upsilon\left(T_{1}\right) \otimes \cdots \otimes \mathbf{A}\left(T_{p}\right)\right)\right) \wedge m_{\partial} z\right) \\
& =\omega_{\partial}\left(\Upsilon_{C}\left(\mathbf{A}\left(T_{1}\right) \otimes \cdots \otimes \mathbf{A}\left(T_{p}\right)\right) \wedge z\right) .
\end{aligned}
$$

\section{A diagrammatic description.}

Broken surfaces are locally flat immersions in the 3-ball $B^{3}$ of disjoint annuli and tori, whose singularities consist of a finite number of circles. Any ribbon tangle in $B^{4}$ can be projected onto a broken surface in a suitable sense. These projections can be viewed as a way to represent ribbon tangles similarly to diagrams in usual knot theory. Conversely, every broken surface is the projection of a ribbon tangle. This gives a correspondence between ribbon tangles and broken surfaces (in fact, of a certain type, called symmetric).

Welded diagrams are a quotient of virtual diagrams under a certain set of moves, see for example [Aud16]. To each welded tangle one may associate a symmetric broken surface diagram, see [Sat00] and [Yaj62]. This define a map Tube sending any welded tangle to the ribbon tangle associated to the symmetric broken surface resulting from the preceding construction.

For general ribbon knotted objects, such as ribbon tangles, ribbon tubes [ABMW17] and knotted spheres [Yan69], the Tube map is well defined and surjective, but its injectivity is still an open question [BD16]. However, this is an isomorphisms on ribbon braids and extended ribbon braids [BH13], [Dam17].

A combinatorial fundamental group can be defined for welded tangle diagrams, with the Wirtinger method (virtual crossings are simply ignored). This group coincides with the fundamental group of the complement of an associated ribbon tangle in $B^{4}$ [Sat00], [Yaj64], [ABMW17]. Then, two welded tangle representing the same ribbon tangle have isomorphic fundamental groups (and this isomorphism sends meridian to meridian).

Let $D$ be the unit disk in $\mathbb{C}$, and for any positive integer $n$, let $x_{1}, \ldots, x_{2 n}$ be a fixed ordered set of points in $\partial D$.

DeFinition 4.1. Let $n$ be a positive integer. A welded tangle on $n$-strands, or welded $n$-tangle is a proper immersion $\tau$ of an oriented 1-manifold in $D$. It consists of some copies of the circle and $n$ copies of the unit interval whose boundary is $\left\{x_{1}, \ldots, x_{2 n}\right\}$. 
The singular set of $\tau$ is a finite number of transversal double points equipped with a partial order on the preimages. By convention, this order is specified by erasing a small neighbourhood of the lowest preimage, or by pointing the crossing if the preimages are not comparable. If the preimages of a crossing are comparable, then the crossing is said classical; otherwise it is said virtual. Moreover, a classical crossing is positive if the basis made of the tangent vectors of the highest and lowest preimages is positive; otherwise, it is negative.

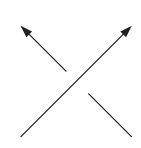

a)

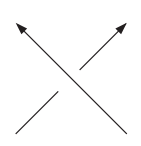

b)

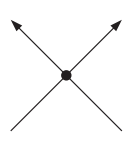

c)

Figure 1. Positive, negative and virtual crossings.

Let $\mu$ be a positive integer. A $\mu$-colored welded tangle is a pair $(\tau, \psi)$ where $\tau$ is a welded tangle and $\psi$ is a map from the set of strands and circles to the set $\left\{t_{1}, \ldots, t_{\mu}\right\}$. Two welded colored tangles are equivalent if they are related by generalized Reidemeister moves (see [Kam07, pp. 445 and 454]), respecting the coloring.

\subsection{Computation of $\mathbf{A}$.}

Let $(\tau, \psi)$ be a $\mu$-colored welded tangle. It decomposes into a finite union of disjoint oriented arcs. Label the crossings with (formal) letters, and each arc with the same letter as the crossing it begins at. If an arc connects points on the border of $\tau$ without meeting any crossing, we use the convention of Figure 3. We construct a matrix $M^{\psi}(\tau)$ with coefficients in $\mathbb{Z}\left[t_{1}^{ \pm 1}, \ldots, t_{\mu}^{ \pm 1}\right]$ where the rows are indexed by crossings (positive, negative and virtual) and points interrupting arcs, and the columns by the arcs.

- Fill row corresponding to each positive and negative crossing as shown in Figure 2,

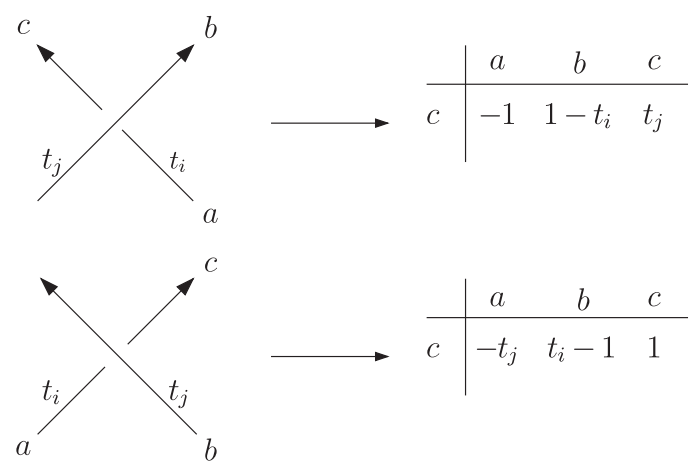

Figure 2. The rule to fill the matrix $M^{\psi}(T)$, where $t_{i}$ and $t_{j}$ are not necessarily different. If $b=a$ or $b=c$ we add the contributions.

- At each point on the diagram, fill the row as shown in Figure 3. 


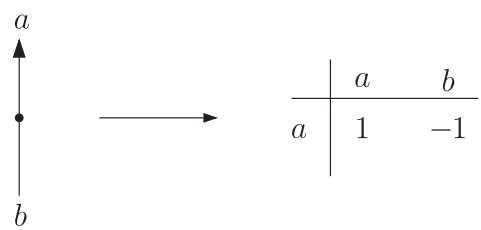

Figure 3. Rule for arcs that don't begin at crossings.

The other entries of the rows are zero. Virtual crossings can be ignored or considered as divided arcs. Notice that, after some Reidemeister moves of type I, one might suppose that there every arc begins at a crossing, and the receipt of Figure 3 becomes useless to construct the matrix $M^{\psi}(\tau)$.

REMARK 4.2. Let $p$ be the number of internal arcs of $\tau$. Since $\tau$ has $2 n \operatorname{arcs}$ connected to the boundary, the total number of arcs is $2 n+p$. One easily observes that the matrix $M^{\psi}(\tau)$ has size $(p+n) \times(p+2 n)$.

Definition 4.3. Let $(\tau, \psi)$ be a $\mu$-colored welded $n$-tangle and $H_{\partial}$ be the module of rank $2 n$ freely generated by the set of marked points $\left\{x_{1}, \ldots, x_{2 n}\right\}$. The invariant $\alpha$ is defined to be

$$
\alpha(\tau, \psi)=\sum_{I}\left|M^{\psi}(\tau)_{I}\right| \cdot x_{I} \in \wedge^{n} H_{\partial},
$$

where the sum is taken for all subset $I \subset\{1, \ldots, 2 n\}$ of $n$ elements, $\left|M^{\psi}(\tau)_{I}\right|$ is the determinant of the $(n+p)$-minor of $M^{\psi}(\tau)$ corresponding to the columns indexed by the internal arcs and the columns relative to the $\operatorname{arcs}$ indexed by $I$, and $x_{I}$ is the wedge product of the generators $x_{i}$ with $i \in I$.

A computation shows that $\alpha(\tau, \psi)$ is invariant by generalized Reidemeister moves, up to multiplication by a unit. Otherwise the invariance is simply a consequence of Theorem 4.6 below.

EXAmple 4.4. Consider the tangle $\tau$ given by one positive crossing, see Figure 1. The matrix $M^{\psi}(\tau)$ coincides with the matrix of Example 2.3. The module $H_{\partial}$ is generated by $x_{1}, \ldots, x_{4}$ and

$$
\alpha(\tau, \psi)=t_{2} x_{3} \wedge x_{4}+x_{2} \wedge x_{3}-t_{2} x_{1} \wedge x_{4}+\left(t_{1}-1\right) x_{1} \wedge x_{3}+x_{1} \wedge x_{2} \in \wedge^{2} H_{\partial} .
$$

Let $\tau$ be a welded tangle. $\pi(\tau)$ denotes the group defined by the Wirtinger method (ignoring the virtual crossings). Then, there is a system of generators of $\pi(\tau)$ in one-toone correspondance with the arcs of $\tau$. In particular, a $\mu$-coloring of a welded tangle $\tau$ can be viewed as a group homomorphism $\psi$ from $\pi(\tau)$ to the free abelian group $\left\langle t_{1}, \ldots, t_{\mu}\right\rangle_{\text {abel }}$ generated by $t_{1}, \ldots, t_{\mu}$.

The following proposition follows directly from the results of Satoh and Yajima [Sat00], [Yaj64]. 
Proposition 4.5. Let $\tau$ be a welded tangle. For any ribbon tangle $T$ such that $T$ is the image of $\tau$ by the Tube map, there is an isomophism

$$
\pi(\tau) \simeq \pi_{1}\left(B^{4} \backslash T\right)
$$

sending arcs of $\tau$ to meridians of $T$.

THEOREM 4.6. Let $\mu$ be a positive integer and $G$ be a free abelian group of rank $\mu$. Let $(\tau, \psi)$ be a $\mu$-colored welded tangle and $(T, \varphi)$ be a $G$-colored ribbon tangle, such that $T$ is the image of $\tau$ by the Tube map. Suppose that there are generators $t_{1}, \ldots, t_{\mu}$ of $G$ such that the following diagram commutes:

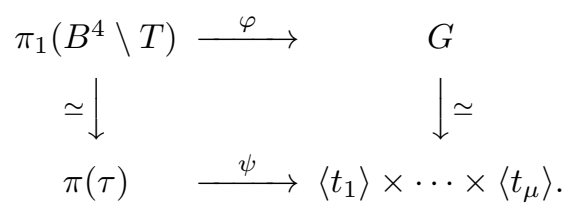

Then,

$$
\mathbf{A}(T, \varphi)=\alpha(\tau, \psi) \in \wedge^{n} H_{\partial}
$$

Welded string links are string links with possibly virtual crossings; for details on these last ones see [KLW01]. Through the Tube map, ribbon tubes can be described by welded string links, see [ABMW17, Section 3.3]. By Theorem 2.10, Proposition 2.9 and Theorem 4.6, the invariant $\alpha$ induces the usual (generalisation of) colored Burau representation - or Gassner, if the coloring is maximal - on (welded) string links [Bar05].

Proof. The points of $\tau \cap \partial B^{2}$ are in one-to-one correspondence with the component of the trivial link $L$ in $T \cap \partial B^{4}$, and $H_{\partial}$ is generated by $x_{1}, \ldots, x_{2 n}$. By Proposition 4.5 and Fox calculus, the matrix $M^{\psi}(\tau)$ is a presentation matrix of the $R$-module $H_{1}^{\varphi}\left(X_{T}, *\right)$, viewed as a $\mathbb{Z}\left[t_{1}^{ \pm 1}, \ldots, t_{\mu}^{ \pm 1}\right]$-module through the choice of generators of $G$. Then $M^{\psi}(\tau)$ is used to compute $\mathcal{A}^{\varphi}$. By definition, for all $I \subset\{1, \ldots, 2 n\}$ with cardinal $n$ :

$$
\omega_{\partial}\left(\mathbf{A}(T, \varphi) \wedge x_{I}\right)=\mathcal{A}^{\varphi}\left(m_{\partial} x_{I}\right)
$$

To calculate $\mathcal{A}^{\varphi}\left(m_{\partial} x_{I}\right)$, we consider the matrix $M^{\psi}(\tau)$, add $n$ row vectors giving the element $m x_{i_{1}}, \ldots, m x_{i_{n}}$ and compute the determinant of the resulting square matrix. Hence adding $m x_{i_{j}}$ corresponds to add the $p+n+j^{\text {th }}$ row $(0, \ldots, 0,1,0, \ldots, 0)$ where 1 is at position $p+j$. We obtain $\mathcal{A}^{\varphi}\left(m_{\partial} x_{I}\right)=\epsilon_{\bar{I}}\left|M^{\psi}(\tau)_{\bar{I}}\right|$, where $\bar{I}$ is the complement of $I$ and $\epsilon_{\bar{I}}$ is the signature of the permutation $I \bar{I}$ (where the elements of $I$ in increasing order are followed by the elements of $\bar{I}$ in increasing order). Finally, we get

$$
\mathbf{A}(T, \varphi)=\sum_{I} \epsilon_{\bar{I}} \cdot \omega_{\partial}\left(\mathbf{A}(T, \varphi) \wedge x_{I}\right) \cdot x_{\bar{I}}=\sum_{I}\left|M^{\psi}(\tau)_{\bar{I}}\right| \cdot x_{\bar{I}}=\alpha(\tau, \psi) .
$$

REMARK 4.7. The invariant $\alpha$ coincides up to a unit with the invariant of virtual tangles introduced by Archibald [Arc10]. It is worth mentioning that using a specific 
canonical choice for the marked points $x_{i}$, her construction is well-defined, not only up to multiplication by a unit (through the multiplication by a correction term).

Proof of Proposition 2.2. As observed in the proof of Theorem 4.6, the matrix $M^{\psi}(\tau)$ is a presentation matrix of $H_{1}^{\varphi}\left(X_{T}, *\right)$, whose size is $(p+n) \times(p+2 n)$, see Remark 4.2. We get a presentation of deficiency $n$.

\subsection{The circuit algebra Weld $_{\mu}$.}

Definition 4.8. A circuit p-diagram consists of the following data:

- The unit disk $D=D_{0}$ in $\mathbb{C}$ together with a finite set of disjoint subdisks $D_{1}, \ldots, D_{p}$ in the interior of $D$. For every $i$ in $\{0, \ldots, p\}$, each $D_{i}$ have $2 n_{i}$ distinct marked points with sign on its boundary (with $n=n_{0}$ ), and a base point $*$ on the boundary of each disk.

- A finite set of embedded oriented arcs whose boundary are marked points in the $D_{i}$. They may cross each other along virtual crossings only. Each marked point is the boundary point of some string — which meets the corresponding disk transversally - and the sign is coherent with the orientation.

Circuit diagrams encode only the matching of marked points, see Figure 4.
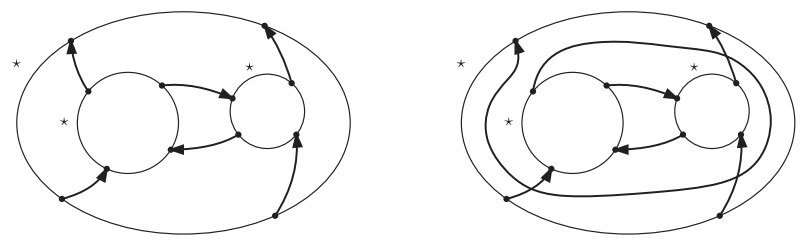

Figure 4. Two equivalent circuit diagrams.

Definition 4.9. Consider a circuit $p^{\prime}$-diagram $P^{\prime}$ and a circuit $p^{\prime \prime}$-diagram $P^{\prime \prime}$ such that $D_{i}^{\prime}$ is a disk of $P^{\prime}$ with $n_{i}^{\prime}=n^{\prime \prime}$, for some $i \in\left\{1, \ldots, p^{\prime}\right\}$. If the signs of the marked points match, we define the diagram $P=P^{\prime} \circ_{i} P^{\prime \prime}$ by rescaling via isotopy the tangle $P^{\prime \prime}$ so that the boundary of $D^{\prime \prime}$ is identified with the boundary of $D_{i}^{\prime}$, and making its marked and base points coincide to those of $D_{i}^{\prime}$. Then $D_{i}^{\prime}$ is removed to obtain $P^{\prime} \circ_{i} P^{\prime \prime}$. This operation is well defined since the starting points eliminate any rotational ambiguity.

A $\mu$-colored circuit diagram is a pair $(P, \psi)$ where $P$ is a circuit diagram and $\psi$ is a map from the set of arcs of $P$ to the set $\left\{t_{1}, \cdots, t_{\mu}\right\}$. Two $\mu$-colored circuit diagrams can be composed once the coloring match on the boundary components. The $\mu$-colored circuit diagrams form an operad $\mathcal{D}_{\mu}$. Let $S=\mathbb{Z}\left[t_{1}^{ \pm 1}, \ldots, t_{\mu}^{ \pm 1}\right]$ be the Laurent polynomial ring. Let $\mathcal{H o m}_{\mu}$ be the operad of tensor products of $S$-modules and $S$-linear maps. The circuit algebra Weld $_{\mu}$ is constructed as a morphism from the operad $\mathcal{D}_{\mu}$ to $\mathcal{H o m}_{\mu}$ as follows.

Consider the unit circle with a base point and a set of marked points $X=$ $\left\{x_{1}, \ldots, x_{2 k}\right\}$, for $k \geq 0$ (with a sign). To this data, we associate the module $\wedge{ }^{k} H_{\partial}$, 
where $H_{\partial}$ is the free $S$-module of rank $2 k$ generated by $X$. Let $(P, \psi)$ be a $\mu$-colored circuit diagram, and $M=\left\{c_{1}, \ldots, c_{q}\right\}$ be the set of curves of $P$. Consider the free module $H$ generated by $M$, and the volume form $\omega: \wedge^{q} H \rightarrow S$ related to this basis. For $i=1, \ldots, p, H_{\partial_{i}}$ denotes the module associated to the boundary circle $\partial D_{i}$ and $H_{\partial}$ the module associated to $\partial D$. Let $m_{i}: H_{\partial_{i}} \rightarrow H$ be the morphims defined by $m_{i}\left(x_{j}\right)=\operatorname{sign}\left(x_{j}\right) c_{j}$ if $x_{j} \in \partial c_{j}$. The mophism $m_{\partial}$ is defined similarly. Let $\omega_{\partial_{i}}$ be the volume form on $H_{\partial_{i}}$ related to the generating system of points of the circle $\partial D_{i}$. Set $m=\otimes_{i}\left(\wedge^{n_{i}} m_{\partial_{i}}\right)$. To the colored diagram $(P, \psi)$ we associate

$$
\gamma_{P, \psi}: \bigotimes_{i=1}^{p} \wedge^{n_{i}} H_{\partial_{i}} \rightarrow \wedge^{n} H_{\partial}
$$

such that, for $x \in \otimes_{i}\left(\wedge^{n_{i}} H_{\partial_{i}}\right)$,

$$
\omega_{C}^{\varphi}\left(m(x) \wedge m_{\partial}(y)\right)=\omega_{\partial}\left(\gamma_{P, \psi}(x) \wedge y\right), \forall y \in \wedge^{n} H_{\partial} .
$$

Then, we can prove Proposition 4.10 below, by repeating the arguments of the proof of Theorem 3.3.

Proposition 4.10. Weld $_{\mu}$ is a circuit algebra.

Note that Weld $_{\mu}$ is similar to half densities introduced by Archibald, see [Arc10] for the definition of half densities and [Dam16] for the explicit correspondence. The morphism $\gamma_{P, \psi}$ could be written as the interior product relative to a subset corresponding to interior arcs of $P$.

The Tube map and the choice of a set of generators $\left\{t_{1}, \ldots, t_{\mu}\right\}$ of $G$ induce a surjective morphism of algebras

$$
\operatorname{Weld}_{\mu} \longrightarrow \mathbf{C o b}_{G}
$$

Given a colored circuit diagram $P$ and a collection of colored tangle diagrams $\tau_{1}, \ldots, \tau_{p}$, one may create a new tangle, if the data on the boundaries and the colorings match, by gluing $\tau_{i}$ into the internal disk $D_{i}$ of $P$. Similarly to Theorem 3.4 , the invariant $\alpha$ commutes with this action of $\mu$-colored circuit diagrams on $\mu$-colored welded tangles. Indeed, one has the following proposition.

Proposition 4.11. Let $(\tau, \psi)$ be the $\mu$-colored welded tangle obtained by gluing the $\mu$-colored welded tangles $\left(\tau_{1}, \psi_{1}\right), \cdots,\left(\tau_{p}, \psi_{p}\right)$ to a $\mu$-colored circuit diagram $(P, \chi)$. The following equality holds:

$$
\alpha(\tau, \psi)=\gamma_{P, \chi}\left(\alpha\left(\tau_{1}, \psi_{1}\right) \otimes \cdots \otimes \alpha\left(\tau_{p}, \psi_{p}\right)\right) \in \wedge^{n} H_{\partial} .
$$

\section{Examples.}

Consider the tangle diagram $\tau$, given in Figure 5 . We let $G=\mathbb{Z}=\langle t>$ and $\psi$ be the coloring sending all arcs of $\tau$ to $t$. In this section, we compute $\alpha(\tau, \psi)$ in different ways. By Theorem 4.6, this computes the value of $\mathbf{A}(T, \varphi)$ the image of $(\tau, \psi)$ by the 


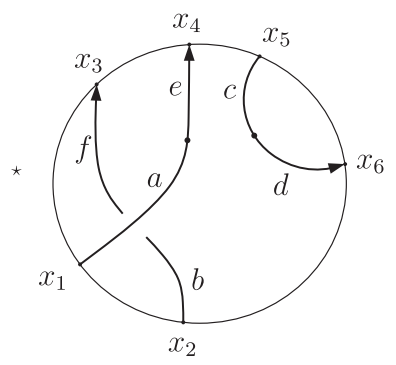

Figure 5. A welded tangle $\tau$.

Tube map.

First, we compute $\alpha(\tau, \psi)$ directly. Label the arcs of $\tau$ with letters $a$ to $f$ as in Figure 5. We obtain the matrix

$$
M^{\psi}(\tau)=\left(\begin{array}{cccccc}
a & b & c & d & e & f \\
0 & 0 & -1 & 1 & 0 & 0 \\
-1 & 0 & 0 & 0 & 1 & 0 \\
1-t & -1 & 0 & 0 & 0 & t
\end{array}\right)
$$

The $\mathbb{Z}\left[t^{ \pm 1}\right]$-module $H_{\partial}$ is free, generated by $x_{1}, \ldots, x_{6}$ and $\alpha(\tau, \psi) \in \wedge^{3} H_{\partial}$ is given by

$$
\begin{aligned}
\alpha(\tau, \psi)= & -x_{1} \wedge x_{2} \wedge x_{5}+x_{1} \wedge x_{2} \wedge x_{6}+x_{2} \wedge x_{5} \wedge x_{4}+(t-1) x_{1} \wedge x_{5} \wedge x_{4} \\
& -t x_{1} \wedge x_{5} \wedge x_{3}+(1-t) x_{1} \wedge x_{6} \wedge x_{4}+t x_{1} \wedge x_{6} \wedge x_{3}+t x_{6} \wedge x_{4} \wedge x_{3} \\
& -x_{2} \wedge x_{6} \wedge x_{4}-t x_{5} \wedge x_{4} \wedge x_{3} .
\end{aligned}
$$

We now consider $(\tau, \psi)$ as the composition of the circuit diagram $(P, \psi)$ with $(\sigma, \psi)$, see Figure 6. We have to compute $\gamma_{P, \psi}: \wedge^{2} H_{\partial_{1}} \rightarrow \wedge^{3} H_{\partial}$ (here $p=1$ ). Let $H$ be the free
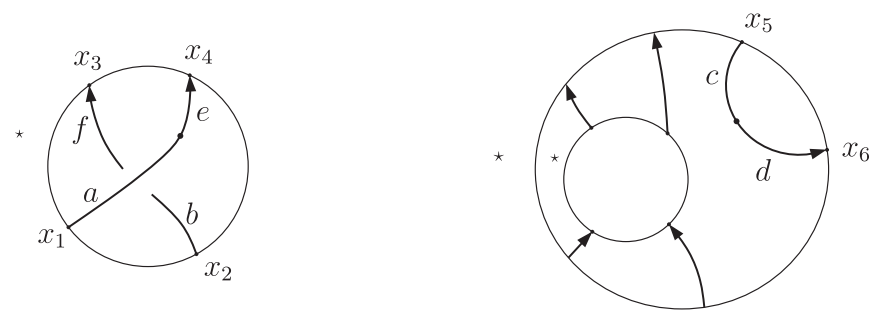

Figure 6. A welded tangle $\sigma$ and a circuit diagram $P$.

module generated by the curves of $\sigma$, labelled $a, b, e, f$. We have that $H_{\partial_{1}}=\left\langle x_{1}, \ldots, x_{4}\right\rangle$ and $H_{\partial}=\left\langle x_{1}, \ldots, x_{6}\right\rangle$. Using the volume form on $H$ related to the choice of the basis $a, b, c, e, d, f$, and the maps induced by the inclusions $m_{1}: H_{\partial_{1}} \rightarrow H$ and $m_{\partial}: H_{\partial} \rightarrow H$, we obtain

$$
\gamma_{C, \psi}\left(x_{i} \wedge x_{j}\right)=x_{i} \wedge x_{j} \wedge\left(x_{6}-x_{5}\right), \forall i, j=1, \ldots, 4,
$$


and

$$
M^{\psi}(\sigma)=\left(\begin{array}{cccl}
a & b & e & f \\
-1 & 0 & 1 & 0 \\
0 & -1 & 1-t & t
\end{array}\right) .
$$

We get

$$
\alpha(\sigma, \psi)=x_{1} \wedge x_{2}+(t-1) x_{1} \wedge x_{4}-t x_{1} \wedge x_{3}+x_{2} \wedge x_{4}-t x_{3} \wedge x_{4} .
$$

The composition $\alpha(\tau, \psi)=\gamma_{P, \psi}(\alpha(\sigma, \psi))$ gives the result. Finally, we consider $\tau$ as the composition of the tangle $\sigma \otimes \beta$ with the circuit $Q$, see Figure 7 . Here $p=2$, and

$$
\gamma_{Q, \psi}: \wedge^{2} H_{\partial_{1}} \otimes H_{\partial_{2}} \rightarrow \wedge^{3} H_{\partial} .
$$
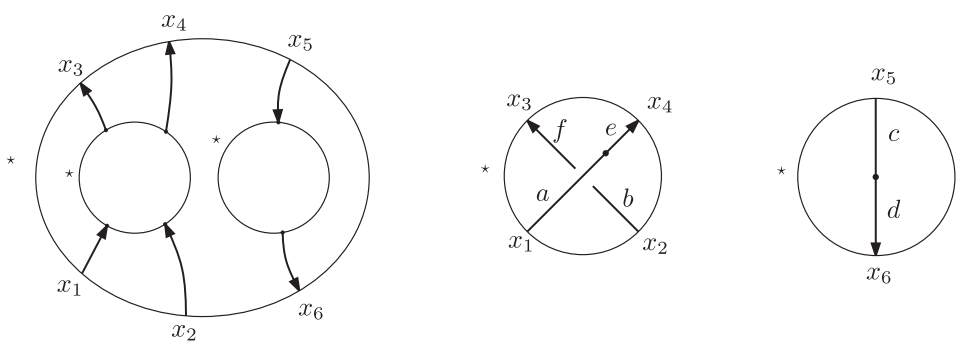

Figure 7. A circuit diagram $Q$ representing a cobordism and two welded tangles $\sigma$ and $\beta$.

As previously $\alpha(\sigma, \psi)=x_{1} \wedge x_{2}+(t-1) x_{1} \wedge x_{4}-t x_{1} \wedge x_{3}+x_{2} \wedge x_{4}-t x_{3} \wedge x_{4} \in \wedge^{2} H_{\partial_{1}}$ and $\gamma(\beta)=x_{6}-x_{5} \in H_{\partial_{2}}$. The composition $\alpha(\tau, \psi)=\gamma_{Q, \psi}(\alpha(\sigma, \psi) \otimes \alpha(\beta, \psi))$ gives the result again.

REMARK 5.1. In the sense of Subsection 2.4, there is a splitting of the tangle $\sigma$ to an (oriented) braid $\sigma_{1}$ in $B_{2}$. We have

$$
M_{0}=\left\langle x_{1}, x_{2}\right\rangle \text { and } M_{1}=\left\langle x_{3}, x_{4}\right\rangle,
$$

and $\alpha(\sigma, \psi) \in \wedge^{2} H_{\partial_{1}} \simeq \wedge^{2}\left(M_{0} \oplus M_{1}\right) \simeq \bigoplus_{k=0}^{2} \wedge^{k} M_{0} \otimes \wedge^{2-k} M_{1}$, similarly to the proof of Theorem 2.10. Then $\alpha(\sigma, \psi)$ decomposes as:

$$
\left(-t x_{3} \wedge x_{4}\right) \oplus\left((t-1) x_{1} \otimes x_{4}-t x_{1} \otimes x_{3}+x_{2} \otimes x_{4}\right) \oplus\left(x_{1} \wedge x_{2}\right) .
$$

Let $\omega_{0}: \Lambda^{2} M_{0} \rightarrow R$ be the volume form related to the basis $\left\langle x_{1}, x_{2}\right\rangle$. For $k=1$, the element $(t-1) x_{1} \otimes x_{4}-t x_{1} \otimes x_{3}+x_{2} \otimes x_{4} \in M_{0} \otimes M_{1}$ induces the morphism $M_{0} \rightarrow M_{1}$ :

$$
x \mapsto(t-1) \omega_{0}\left(x \wedge x_{1}\right) \cdot x_{4}-t \omega_{0}\left(x \wedge x_{1}\right) \cdot x_{3}+\omega_{0}\left(x \wedge x_{2}\right) \cdot x_{4} .
$$

The image of $x_{1}$ is $x_{4}$ and the image of $x_{2}$ is $(1-t) x_{4}+t x_{3}$. This corresponds to the Burau representation. Similalrly, the other values of $k$ give $k^{\text {th }}$-exterior powers of Burau, 
up to a sign.

Acknowledgements. The authors thank B. Audoux for accurate comments on the manuscript. They thank P. Bellingeri, J.-B. Meilhan and E. Wagner for helpful comments, and G. Massuyeau who brought our attention to the similarities between Archibald's invariant and the functor constructed by Bigelow, Cattabriga and the second author.

\section{References}

[ABMW17] B. Audoux, P. Bellingeri, J.-B. Meilhan and E. Wagner, Homotopy classification of ribbon tubes and welded string links, Ann. Sc. Norm. Super. Pisa Cl. Sci. (5), 17 (2017), 713-761.

[Arc10] J. Archibald, The Multivariable Alexander Polynomial on Tangles, Ph.D. thesis, University of Toronto (Canada), 2010.

[Aud16] B. Audoux, On the welded tube map, Knot theory and its applications, Contemp. Math., 670, Amer. Math. Soc., Providence, RI, 2016, 261-284.

[Bar05] V. G. Bardakov, Extending representations of braid groups to the automorphism groups of free groups, J. Knot Theory Ramifications, 14 (2005), no. 8, 1087-1098.

[BCF15] S. Bigelow, A. Cattabriga and V. Florens, Alexander representation of tangles, Acta Math. Vietnam., 40 (2015), 339-352.

[BD16] D. Bar-Natan and Z. Dancso, Finite type invariants of $w$-knotted objects I: $w$-knots and the Alexander polynomial, Algebr. Geom. Topol., 16 (2016), 1063-1133.

[BH13] T. E. Brendle and A. Hatcher, Configuration spaces of rings and wickets, Comment. Math. Helv., 88 (2013), 131-162.

[Big12] S. Bigelow, A diagrammatic Alexander invariant of tangles, J. Knot Theory Ramifications, 21 (2012), no. 8, 1250081.

[BNS13] D. Bar-Natan and S. Selmani, Meta-monoids, meta-bicrossed products, and the Alexander polynomial, J. Knot Theory Ramifications, 22 (2013), no. 10, 1350058.

[CT05] D. Cimasoni and V. Turaev, A lagrangian representation of tangles, Topology, 44 (2005), $747-767$.

[Dam16] C. Damiani, The topology of loop braid groups: applications and remarkable quotients, Ph.D. thesis, Université de Caen Normandie, 2016.

[Dam17] C. Damiani, A journey through loop braid groups, Expo. Math., 35 (2017), 252-285.

[FM15] V. Florens and G. Massuyeau, A functorial extension of the abelian Reidemeister torsions of threemanifolds, Enseign. Math., 61 (2015), 161-210.

[Fox73] R. H. Fox, Characterizations of slices and ribbons, Osaka J. Math., 10 (1973), 69-76.

[Jon99] V. F. R. Jones, Planar algebras, I, ArXiv Mathematics e-prints (1999).

[Kam07] S. Kamada, Braid presentation of virtual knots and welded knots, Osaka J. Math., 44 (2007), 441-458.

[Ken13] K. G. Kennedy, A Diagrammatic Multivariate Alexander Invariant of Tangles, Ph.D. thesis, University of California, Santa Barbara, 2013.

[KLW01] P. Kirk, C. Livingston and Z. Wang, The Gassner representation for string links, Commun. Contemp. Math., 3 (2001), 87-136.

[Les98] C. Lescop, A sum formula for the Casson-Walker invariant, Invent. Math., 133 (1998), 613-681.

[Pol10] M. Polyak, Alexander-Conway invariants of tangles, ArXiv e-prints (2010).

[Sar15] A. Sartori, The Alexander polynomial as quantum invariant of links, Ark. Mat., 53 (2015), 177-202.

[Sat00] S. Satoh, Virtual knot presentation of ribbon torus-knots, J. Knot Theory Ramifications, 9 (2000), no. 4, 531-542.

[Yaj62] T. Yajima, On the fundamental groups of knotted 2-manifolds in the 4-space, J. Math. Osaka City Univ., 13 (1962), 63-71.

[Yaj64] T. Yajima, On simply knotted spheres in $R^{4}$, Osaka J. Math., 1 (1964), 133-152. 
[Yan69] T. Yanagawa, On ribbon 2-knots, II, The second homotopy group of the complementary domain, Osaka J. Math., 6 (1969), 465-473.

[Zib16] C. Zibrowius, On a polynomial Alexander invariant for tangles and its categorification, ArXiv e-prints (2016).

\section{Celeste DAMIANI}

Department of Mathematics

Osaka City University

Sugimoto, Sumiyoshi-ku

Osaka 558-8585, Japan

E-mail: celeste.damiani@math.cnrs.fr

\section{Vincent FLORENS}

Laboratoire de Mathématiques et leurs applications UMR CNRS 5142

Université de Pau et des Pays de l'Adour

Avenue de l'Université

BP 115564013 PAU Cedex, France

E-mail: vincent.florens@univ-pau.fr 